\title{
Actualización: \\ Deterioro cognitivo leve y deterioro cognitivo sin demencia
}

Mild cognitive impairment and cognitive impairment without dementia

Chertkow H y col. CMAJ 2008; 178 (10):1273-85.

\begin{abstract}
Resumen
El presente material resume en castellano las recomendaciones sobre el abordaje diagnóstico y terapéutico del deterioro cognitivo leve y el deterioro cognitivo sin demencia; generadas en marzo de 2006 y recientemente publicadas por el Tercer Consenso Canadiense para el Diagnóstico y Tratamiento de la Demencia.

\section{Abstract}

This material summarizes the recommendations about diagnosis and treatment of Mild Cognitive Impairment, and Cognitive Impairment without Dementia, developed in March 2006 and recently published by the Third Canadian Consensus for the Diagnosis and Treatment of Dementia.
\end{abstract}

\section{Introducción}

Entre un 25 y un $75 \%$ de los adultos mayores se queja del funcionamiento de su memoria. Si bien la mayoría no presenta deterioro cognitivo, muchos de ellos consultan por temor a que dicho problema corresponda al comienzo de una enfermedad de Alzheimer (EA) o algún otro tipo de demencia ${ }^{1,2}$.

El deterioro cognitivo leve (DCL) y el deterioro cognitivo sin demencia o "no demencia" (DCND) son conceptos relativamente nuevos que intentan incluir a aquellos individuos que presentan alteraciones cognitivas que no llegan a ser lo suficientemente graves como para definir un síndrome demencial. Las controversias en su definición hacen que su incidencia y prevalencia varíen considerablemente de acuerdo a los criterios utilizados ${ }^{3,4}$. Además, teniendo en cuenta que muchos de estos pacientes evolucionarán a una demencia, muchos expertos consideran a estas entidades como etapas prodrómicas de aquella ${ }^{5,6}$.

Debido a la creciente incorporación de nueva información relacionada al deterioro cognitivo/demencia, la misma fue actualizada en marzo de 2006 en el Tercer Consenso Canadiense para el Diagnóstico y el Tratamiento de la Demencia, cuya edición previa databa de 1999. Siguiendo criterios metodológicos preestablecidos se revisó, calificó y sintetizó la información disponible de los últimos diez años y se generaron una serie de recomendaciones calificadas según el nivel de evidencia que las respalda ${ }^{7}$. En este resumen que publicamos en EVIDENCIA se incluyen las recomendaciones específicamente referidas al manejo del DCL/DCND.

\section{Definiciones}

Como dijimos previamente, el concepto DCL/DCND incluye a aquellos pacientes que se encuentran en la zona gris entre la demencia y la "normalidad", entendiendo a esta última como la ausencia de alteraciones cognitivas s,8,9. $^{3,9}$

Deterioro cognitivo leve: este término se aplica a quienes presentan alteración en la memoria, sin una importante disfunción en sus actividades de la vida cotidiana (DCL amnésico). Sin embargo, no existe mucho consenso para definir que significa una "importante disfunción" y cuan atribuible sería dicha disfunción a dicho DCL. En este sentido vale mencionar que algunos trabajos han encontrado que hasta el $31 \%$ de los pacientes clasificados o definidos como DCL presentan trastornos funcionales de base ${ }^{10}$.

Deterioro cognitivo "no demencia": se trata de un concepto un poco menos específico que el anterior ya que hace referencia a un grupo similar de pacientes, pero sin determinar el grado de deterioro funcional presente ${ }^{11}$.

Se recomienda a los profesionales que estén familiarizados con ambos conceptos (se resumen en la tabla 1) ya que dado que la existencia de cualquiera de las dos situaciones expresa un alto riesgo de progresión a demencia (nivel de evidencia 3) es común que sean utilizados en forma indistinta. De aquí en adelante al referirnos a DCL haremos referencia a ambos conceptos.

Tabla 1: características diferenciales del deterioro cognitivo leve y del deterioro cognitivo sin demencia.

\begin{tabular}{l|c|c}
\multicolumn{1}{c|}{ Característica } & Deterioro cognitivo Leve & Deterioro cognitivo sin demencia \\
\hline Queja de memoria reportada por el paciente o un familiar & Si & No necesariamente \\
\hline $\begin{array}{l}\text { Evidencia objetiva de deterioro de memoria u otro dominio cognitivo basada en una } \\
\text { evaluación neuropsicológica estandarizada }\end{array}$ & $\begin{array}{c}\text { También puede basarse en } \\
\text { una batería breve de pruebas }\end{array}$ & $\mathrm{Si}$ \\
\hline Preservación de actividades básicas de la vida diaria & Siempre \\
\hline Cumplimiento de criterios de demencia & $\mathrm{Si}$ & No especificado \\
\hline Historia de declinación funcional desde un nivel previamente normal & No necesariamente \\
\hline
\end{tabular}

\section{Evaluación diagnóstica}

El diagnóstico de DCL es eminentemente clínico. Para ello se recomienda evaluar al paciente como si presentara un síndrome demencial: con una anamnesis y un examen físico apropiados, con pruebas de evaluación cognitiva, con un laboratorio y con una imagen cerebral. En este sentido, la meta es objetivar el deterioro cognitivo y a su vez, identificar posibles causas secundarias ${ }^{12}$. 


\section{Objetivación del deterioro cognitivo}

El Mini Mental State Examination (Sigla en inglés, MMSE) es una de las baterías breves mas difundidas en la atención primaria pero resulta poco sensible para detectar alteraciones cognitivas leves. Por lo tanto, se recomienda el uso alternativo de dos pruebas: el DemTect o el Montreal Cognitive Asessment $(\mathrm{MoCA})^{13}$, que amplían los dominios evaluados por el MMSE y pueden asimismo ser administradas en el consultorio (ninguna de las dos aún validadas al español).

Estas mismas pruebas se han propuesto como instrumentos de rastreo del DCL, con resultados prometedores ${ }^{14,15}$. Cuando persisten dudas diagnósticas se recomienda la realización de baterías neuropsicológicas más exhaustivas.

\section{Determinación de la presencia de deterioro funcional}

Como se menciono previamente, un punto saliente en el diagnóstico de DCL tiene que ver con la ausencia de deterioro funcional importante ${ }^{11}$, siendo este punto muchas veces de difícil definición.

Las preguntas más sensibles que podrían detectar dichas alteraciones parecerían ser:

¿Mantiene sus hobbies o pasatiempos?

¿Es capaz de manejar asuntos financieros complejos? ¿Es capaz de manejar nuevas herramientas?

¿Se olvida el día y el año?

¿Está reiterativo?

\section{Pesquisa de comorbilidades agravantes}

Existe un número considerable de situaciones comórbidas que pueden generar o exacerbar un déficit cognitivo y que deben ser consideradas al momento de establecer diagnósticos diferenciales. Mencionamos como ejemplo a la apnea obstructiva ${ }^{16}$, a otros trastornos del sueño $0^{17}$, a los efectos adversos farmacológicos y al aislamiento social ${ }^{18}$.

El diagnóstico concurrente de depresión no tratada o subtratada representa un punto de controversia importante. Si bien algunos autores sugieren que debe considerarse un criterio de exclusión; otros lo consideran un signo pronóstico, dado el hallazgo concurrente de depresión en hasta el $60 \%$ de los pacientes con DCL que evolucionan luego a Enfermedad de Alzheimer ${ }^{19}$

\section{Riesgo de progresión a demencia}

En la mayoría de los estudios clínicos, entre el 40 y el $80 \%$ de los pacientes con DCL desarrollan EA a los cinco años. La tasa de conversión anual ronda entre el 10 y el $15 \%{ }^{67}$.

Entonces: ¿es posible identificar a los pacientes que desarrollarán EA? Si bien se han propuesto numerosos marcadores (beta amiloide y proteína tau en el líquido cefaloraquídeo, homocisteinemia, dosaje de interleuquina 6, APOE, hemoxigenasa 1; medición del volumen hipocampal por neuroimágenes, tomografia por emisión de positrones) la mayoría de estos, provienen de trabajos pequeños con poco seguimiento. Por lo tanto, todavía existe evidencia insuficiente como para generalizar una recomendación sobre su uso.

Dado el mayor riesgo de progresión a demencia en los pacientes con $\mathrm{DCL}$, se recomienda su monitoreo estrecho (recomendación tipo B) por ejemplo a través de una reevaluación clínica anual y eventual reevaluación neuropsicológica.

\section{Tratamiento}

\section{Estimulación cognitiva y actividad física}

La evidencia actual es insuficiente para concluir que intervenciones como la estimulación cognitiva sean beneficiosas para prevenir la progresión a demencia ${ }^{20}$ (recomendación tipo $\mathrm{C}$ y grado de evidencia tipo 1). En este aspecto, tampoco existe evidencia suficiente para recomendar un programa específico de actividad física (recomendación tipo $\mathrm{C}$ y grado de evidencia tipo 3). Sin embargo, ambas actividades deberían ser promovidas en todas las personas mayores, tengan o no, trastornos de la memoria (recomendación tipo $\mathrm{B}$, grado de evidencia tipo 2).

Manejo de los factores de riesgo cardiovascular

Los pacientes con DCL no escapan a las recomendaciones generales de rastreo de los diferentes factores de riesgo cardiovascular ${ }^{21,22}$ (FRC) (recomendación tipo $B$, grado de evidencia tipo 2). Por ejemplo, el tratamiento de la hipertensión sistólica en los adultos mayores reduce el riesgo de deterioro cognitivo asociado a enfermedad cerebrovascular; recomendándose mantener la TA sistólica por debajo de $140 \mathrm{mmHg}$ (recomendación tipo $A$, evidencia grado 1). Si bien no hay evidencia de la superioridad de algún agente anti-hipertensivo sobre el resto, podrían considerarse como primera elección de tratamiento los bloqueantes de los canales de calcio y los inhibidores de la enzima convertidota de angiostensina (recomendación tipo B, evidencia grado 1).

Por otro lado, no existe evidencia actual que apoye el uso de aspirina para tratar específicamente la demencia asociada a la enfermedad cerebrovascular (recomendación tipo C, evidencia grado 3 ). Sin embargo la aspirina -u otras terapias antiplaquetarias- deberían usarse para la prevención de accidente cerebrovascular isquémico recurrente (recomendación tipo A, evidencia grado 1$)^{23,24}$.

\section{Tratamiento farmacológico específico}

No existe suficiente evidencia actual para recomendar el uso de anticolinesterásicos ${ }^{25}$ en pacientes con DCL (recomendación tipo $\mathrm{C}$, evidencia grado 1). Por otro lado, actualmente existe suficiente evidencia para no recomendar el uso de anti inflamatorios no esteroides ${ }^{26}$ (AINE) la terapia de reemplazo hormonal ${ }^{27}$, el Ginkgo biloba ${ }^{28}$ y la vitamina $E^{29}$ para el tratamiento del DCL (fuerza de la recomendación tipo D, evidencia grado 1).

\section{Consejo médico y educación}

Si bien no hay estudios que evalúen el efecto psicológico o social del diagnóstico de "deterioro cognitivo leve", la impresión general, es que resulta más beneficioso brindarlo, que decir al paciente:

“usted está normal" o "no sé muy bien lo qué le pasa".

Según la evidencia actual no seria de buena práctica afirmar que los pacientes con DCL presentan "signos de demencia"; ni tampoco ofrecerles tratamiento como si su síndrome fuera equivalente a la demencia (fuerza de la recomendación tipo $\mathrm{C}$ y evidencia grado 2).

Palabras claves: deterioro cognitivo, definición, diagnóstico, tratamiento. Key words: mild cognitive impairment, definition, diagnosis, treatment. 


\section{Comentario}

El envejecimiento poblacional hace imperioso que los profesionales que trabajan en atención primaria se familiaricen con el diagnóstico de DCL ya que la población asistida presentará cada vez mayor prevalencia de este tipo de trastornos debido al envejecimiento poblacional.

Todavía queda un amplio campo por investigar sobre sus factores de riesgo, y sobre la eficacia de las intervenciones farmacológicas y no farmacológicas.

También se requieren más estudios que evalúen la complejidad de los componentes involucrados en este diagnóstico, así como las herramientas clínicas disponibles para realizarlo. Respecto del rastreo de DCL, si bien ya contamos con herramientas que poseen buenas características operativas para su diagnóstico, todavía no contamos con evidencia que permita saber si la precocidad del mismo cambiará algún resultado como el pronóstico de dicha entidad, la calidad de vida de los convivientes, etc.

\section{Conclusión de la comentadora}

A modo de resumen podríamos plantear que al momento actual, existe suficiente evidencia que avala no usar AINE, vitamina $E$, ginkgo biloba, terapia de reemplazo estrogénico y AAS para el tratamiento del DCL, siendo ésta todavía insuficiente respecto del uso de los inhibidores de la colinesterasa.

Por otro lado, existe suficiente respaldo bibliográfico para recomendar en forma general la promoción de un estilo de vida saludable en todos los adultos mayores (tengan o no DCL); lo que incluye la recomendación de actividad física, la estimulación cognitiva, las actividades placenteras y el tratamiento adecuado de los factores de riesgo cardiovascular.

Mariela Gonzalez Salvia [ Servicio de Clínica Médica del Hospital Italiano de Buenos Aires, Sección de Geriatría. mariela.gonzalez@hospitalitaliano.org.ar ]

González Salvia M. Deterioro cognitivo leve y deterioro cognitivo sin demencia. Evid. actual. práct. ambul; 11(6): 179-182, Nov-Dic.2008. Comentado de: Chertkow H. Diagnosis and treatment of dementia: introduction. Introducing a series based on the Third Canadian Consensus Conference on the Diagnosis and Treatment of Dementia. CMAJ2008; 178:316-21. PMID: 18458258.

Disponible en URL: http://www.cmaj.ca/cgi/content/abstract/178/10/1273.

\section{Referencia}

1. Jonker C y col. Are memory complaints predictive for dementia? A review of clinical and population-based studies. Int J Geriatr Psychiatry 2000; $15: 983-91$.

2. Hanninen T y col. Prevalence of mild cognitive impairment: a population-based study in elderly subjects. Acta Neurol Scand 2002;106:148-54.

3. Petersen R y col. Mild cognitive impairment: clinical characterization and outcome. Arch Neurol1999;56:303-8.

4. Petersen R y col. Practice parameter: early detection of dementia: mild cognitive impairment (an evidence-based review). Report of the Quality Standards Subcommittee of the American Academy of Neurology. Neurology 2001;56:1133-42.

5 . Fisk J y col. Variations in case definition affect prevalence but not outcomes of mild cognitive impairment. Neurology2003;61:1179-84

6. Daly E y col. Predicting conversion to Alzheimer disease using standardized clinical information. Arch Neurol2000;57:675-80.

7. Chertkow H. Diagnosis and treatment of dementia: introduction. Introducing a series based on the Third Canadian Consensus Conference on the Diagnosis and Treatment of Dementia. CMAJ2008;178:316-21.

8. Smith A y col. Use of structural imaging to study the progression of Alzheimer's disease. Br Med Bull1996;52: 575-86.

9. Bennett D y col. Neuropathology of older persons without cognitive impairment from two community-based studies. Neurology 2006;66:1837-44.

10. Artero S y col. Disability and mild cognitive impairment: a longitudinal population-based study. Int J Geriatr Psychiatry2001;16:1092-7.

11. Feldman $\mathrm{H}$ y col. A Canadian cohort study of cognitive impairment and related dementias (ACCORD): study methods and baseline results. Neuroepidemiology2003;22:265-74.

12. Feldman H y col. Diagnosis and treatment of dementia: 2. Diagnosis. CMAJ 2008;178:825-36.

13. Nasreddine Z. The Montreal Cognitive Assesment.[pagina principal de internet]. Disponible en: http://www.mocatest.org/default.asp (ultimo acceso 09/11/2008).

14. Petersen R y col. Mild cognitive impairment: clinical characterization and outcome. Arch Neurol1999;56:303-8.

15. Nasreddine Z y col. The Montreal Cognitive Assessment, MoCA: a brief screening tool for mild cognitive impairment. J Am Geriatr Soc 2005;53:695-9.

16. Bliwise D. Is sleep apnea a cause of reversible dementia in old age? J Am Geriatr Soc1996;44:1407-9.

17. Riemann D y col. Cholinergic neurotransmission, REM sleep and depression. J Psychosom Res1994;38(Suppl 1):15-25

18. Fratiglioni $L$ y col. Influence of social network on occurrence of dementia: a community-based longitudinal study. Lancet2000;355:1315-9.

19. Visser P y col. Distinction between preclinical Alzheimer's disease and depression. J Am Geriatr Soc2000;48:479-84.

20. Wilson R y col. Participation in cognitively stimulating activities and risk of incident Alzheimer disease. JAMA2002;287:742-8.

21. Ott A y col. Diabetes mellitus and the risk of dementia: the Rotterdam Study. Neurology1999;53:1937-42.

22. Elias M y col. Lower cognitive function in the presence of obesity and hypertension: the Framingham heart study. Int J Obes Relat Metab Disord2003:27:260-8.

23. Starr $\mathrm{J}$ y col. The effects of antihypertensive treatment on cognitive function: results from the HOPE study. J Am Geriatr Soc1996; 44:411-5.

23. Starr $\mathrm{J} y$ col. The effects of antihypertensive treatment on cognitive function: results from the HOPE study. J Am Geriatr Soc1996; 44:411-5.
24. Shepherd J y col. Pravastatin in elderly individuals at risk of vascular disease (PROSPER): a randomised controlled trial. Lancet2002; 360:1623-30.

25. Salloway S y col. Efficacy of donepezil in mild cognitive impairment: a randomized placebo-controlled trial. Neurology2004;63:651-7.

26. Thal $L$ y col. A randomized, double-blind, study of rofecoxib in patients with mild cognitive impairment. Neuropsychopharmacology2005;30:1204-15

27. Shumaker $S$ y col. Estrogen plus progestin and the incidenceof dementia and mild cognitive impairment in postmenopausal women: theWomen's Health Initiative Memory Study: a randomized controlled trial. JAMA 2003;289:2651-62.

28. Van Dongen M y col. Ginkgo for elderly people with dementia and age-associated memory impairment: a randomized clinical trial. J Clin Epidemiol2003;56:367-76.

29. Petersen R y col. Vitamin E and donepezil for the treatment of mild cognitive impairment. N Engl J Med2005;352:2379-88. 